\title{
El fortalecimiento del instituto federal de TELECOMUNICACIONES COMO SEÑAL DEL NUEVO EQUILIBRIO DEL PODER EN EL ESTADO MEXICANO.
}

\author{
MARCo ANTONIO ZEIND ChÁVEZ ${ }^{1}$
}

Resumen: Dentro de la nueva conformación del Derecho en el siglo XXI, las modificaciones constitucionales en áreas específicas (tales como lo son las telecomunicaciones) son relevantes en la nueva forma de comprender y desarrollar los mecanismos de control democrático que existe entre las instituciones que los regulan.

La sentencia que resuelve el amparo 1100/2015, es de vital importancia en el desarrollo de la investigación y consolidación de los Organismos Constitucionales Autónomos (OCAs).

Han sido estos entes públicos los que han dado el giro de tuerca en la forma de abordar la división de poderes y las facultades de los Poderes Ejecutivo y Legislativo, los cuales se ven confrontados por la capacidad técnica y especializada de algunos OCAs. De ahí surgió la necesidad e inquietud de analizar una sentencia que marca un punto de quiebre no sólo para los mismos OCAs; sino en asuntos tan específicos como son las tarifas de interconexión.

PALABRAS ClAVE: división de poderes, organismos, constitucionales autónomos (OCAs), leyes y reglamentos, Suprema Corte de Fusticia de la Nación $(S C \mathcal{J} \mathcal{N})$, amparo, sentencia.

ABSTRACT: Within the new conformation of the Law in the 21st century, the constitutional amendments in specific fields (such as telecommunications) are relevant in the new way of understanding and developing new mechanisms of democratic control of institutions that regulate them. The resolution 1100/2015 that sets forth the legal protection is utterly relevant in the development of the investigation and consolidation of the Autonomous Constitutional Entities (OCAs). These public entities have given a twist in the way of approaching the separation of powers and the authorities

\footnotetext{
1 Facultad de Derecho, UNAM.
} 
of the Executive and Legislative Powers, that are confronted by the technical and specialised capacities of some OCAs. This is the rationale behind the concern and necessity of analysing such resolution causing a breaking point for both of them, and also for specific areas such as the interconnection rates.

Keywords: Separation of powers, constitutional autonomous entities, laws and regulations, Mexican Supreme Court of Fustice, amparo, resolution.

Sumario: I. La división del poder en México; II. El Instituto Federal de Telecomunicaciones (IFT); III. Aspectos para considerar aplicables al caso concreto; IV. Determinación como AEP al grupo de interés económico del que forman parte América Móvil. S.A.B. de G.V., Teléfonos de México. S.A.B. de G.V., Teléfonos del Noroeste. S.A. de C.V., Radiomóvil Dipsa S.A.B. de C.V., Grupo Carso S.A. B. de G.V., y Grupo Financiero lnbursa. S.A.B. de G.V. (TELMEX y TELCEL) y MRA impuestas; V. Tarifa Cero en Interconexión, el ACUERDO P/IFT/260314/17y la LFTR. La clave; VI. La consolidación del IFT; VII. Conclusiones.

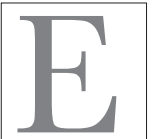

1 pasado 16 de agosto de 2017 la Suprema Corte de Justicia de la Nación (SCJN) emitió una resolución mediante la cual otorgó un amparo a Radiomóvil Dipsa, S.A. de C.V. (comúnmente conocido como Telcel) en contra de la prohibición de cobrar tarifas de interconexión a las empresas que compiten con ella en el sector de la telefonía, tanto fija como celular. Con el fin de entender el fondo y los alcances de esta determinación del Máximo Tribunal, se considera importante aclarar algunos conceptos que serán de utilidad para el lector, para así, posteriormente profundizar en el caso específico.

\section{LA división del poder en México}

La Constitución Política de los Estados Unidos Mexicanos (Constitución) señala en su artículo 49 que el Supremo Poder de la Federación se divide para su ejercicio en Legislativo, Ejecutivo y Judicial. Lo anterior, en estricta consonancia con lo señalado por teóricos clásicos que lograron crear una doctrina de tal envergadura que 
ha logrado permanecer prácticamente intocada desde su génesis, consiguiendo que hasta el momento ejemplos como el de nuestra Constitución sigan estableciendo lo estipulado por dicha doctrina. No obstante, la realidad constitucional y legal actual han rebasado y confrontado de manera clara esta división, con lo que se tiene un ejemplo más de cuando la realidad se adelanta al orden jurídico y entonces obliga a este último a modificarse para adecuarse a la primera, esto con el fin primordial de intentar con ello seguir propugnando la convivencia armónica de la sociedad, como originaria y destinataria de su existencia, confirmándose de esta manera que el Derecho se adecua a la velocidad de la realidad, y no viceversa.

El desafío aludido comenzó a presentarse desde 1993, año en el cual el gobierno mexicano decidió trastocar por primera vez desde el plano de los hechos la teoría clásica de la división de poderes: se trató del momento en el cual se dotó de autonomía a través de la reforma al artículo 28 constitucional al Banco de México (BANXI$\mathrm{CO})$, el cual es el banco central del Estado Mexicano. Básicamente, al dotar de autonomía constitucional a un ente público, se genera que dicha institución deje de encontrarse adscrita al poder Legislativo, Ejecutivo o Judicial o, incluso, puede darse el caso que desde su nacimiento no dependa de ninguno de estos. Incluso, el hecho de que una institución pública esté dotada de autonomía representa el abandono a cualquier posibilidad de sometimiento a cualquier de los poderes, colocándose en un plano de igualdad tanto desde la perspectiva jurídica como de la política o, en otras palabras, la existencia de una paridad jerárquica en los casos en donde dicha autonomía se logre completamente.

Es menester dejar claro que si bien en México los Organismos Constitucionales Autónomos (OCAs) vieron su nacimiento en la recta final del siglo XX, "en otros países surgen luego de la Segunda Guerra Mundial, aunque fueron ya teorizados por Georg Jellinek y por Santi Romano desde finales del siglo XIX. La existencia de dichos órganos supone un enriquecimiento de las teorías clásicas de 
la división de poderes que postulaban que dentro de un Estado solamente había tres funciones: la legislativa, la ejecutiva y la judicial". ${ }^{2}$ Aunque este autor deja claro que este tipo de entes son una realidad alrededor del mundo desde hace varios años, se nota cierta timidez al momento de considerar que su existencia supone un "enriquecimiento de las teorías clásicas de la división de poderes"; el solo hecho de que su nacimiento se pueda traducir en extraer del ámbito de competencia del poder Legislativo, Ejecutivo o Judicial alguna atribución, podría generar el efecto contrario, traduciéndose en un debilitamiento de la división de poderes tal como se entiende al día de hoy.

El desarrollo de los OCAs ha quedado claro desde el momento en que en los últimos años su existencia ha ido en aumento en México. Como resultado de la importancia que gradualmente han ido ganando, la SCJN ha detallado cuáles son sus características principales por medio de diversas jurisprudencias, ${ }^{3}$ siendo aquéllas las siguientes:

1. Surgen bajo una idea de equilibrio constitucional basada en los controles de poder.

2. La creación de este tipo de órganos no altera o destruye la teoría tradicional de la división de poderes.

3. Son organismos autónomos cuya actuación no está sujeta ni atribuida a los depositarios tradicionales del poder público (poderes legislativo, ejecutivo y judicial).

2 Caballero, José Antonio, Et al, El futuro del Instituto Federal de Acceso a la Información Pública y Protección de Datos Personales. Consideraciones sobre su autonomía Constitucional, UNAM/Instituto de Investigaciones Jurídicas, 2012, p.8

${ }^{3}$ Tesis P. /J. 20/2007, Semanario Judicial de la Federación y su Gaceta, Novena Época, t. XXV, mayo de 2007, p. 1647 y Tesis P. /J. 12/200, Semanario Judicial de la Federación y su Gaceta, Novena Época, t. XXVII, febrero de 2008, p. 1871 . Consultadas el 7 de septiembre de 2017. 
4. Se le han conferido funciones estatales específicas, con el fin de obtener una mayor especialización, agilización, control y transparencia para atender eficazmente las demandas sociales.

5. Su misión principal radica en atender necesidades torales tanto del Estado como de la sociedad en general, conformándose como nuevos organismos que se encuentran a la par de los órganos tradicionales.

6. Aunque no existe algún precepto constitucional que regule su existencia, deben cumplir con lo siguiente:

a) Estar establecidos y configurados directamente en la constitución.

b) Mantener con los otros órganos del Estado relaciones de coordinación.

c) Contar con autonomía e independencia funcional y financiera.

d) Atender funciones primarias u originarias del Estado que requieran ser eficazmente atendidas en beneficio de la sociedad.

Como es notable, gracias a estas tesis de jurisprudencia se brinda una idea más cercana de lo que esta figura jurídica implica, aunque se persiste en el hecho de aclarar que la creación de los OCAs no altera o destruye la teoría tradicional de la división de poderes.

\section{il. El Instituto Federal de Telecomunicaciones (IFT)}

El IFT tuvo como antecedente inmediato a la Comisión Federal de Telecomunicaciones (COFETEL), la cual se trató de un órgano desconcentrado creado en el decreto publicado en el Diario Oficial de la Federación (DOF) el 9 de agosto de 1996. Este órgano contaba con autonomía técnica y operativa, el cual tendría la organización y 
facultades necesarias para regular y promover el desarrollo eficiente de las telecomunicaciones en el país.

Apenas iniciando el sexenio del presidente Peña Nieto, se decidió llevar a cabo una negociación de alto nivel con los partidos políticos con mayor peso electoral (PAN, PRD y PRI) para lograr un acuerdo del cual se conocería su existencia apenas al comienzo de su administración, el conocido como "Pacto por México", considerado como la plataforma de lanzamiento para el proceso reformador anunciado por el Jefe de Estado Mexicano. Así, con la firma del mismo, se buscó que las cúpulas partidistas realizaran una serie de reformas constitucionales y legales consideradas necesarias en los últimos años, las conocidas como reformas estructurales. Entre los acuerdos a los que se llegó estuvieron los signados "para el crecimiento económico, el empleo y la competitividad", contando entre ellos con aquel cuya finalidad fue "garantizar el acceso equitativo a telecomunicaciones de clase mundial". Es de ahí de donde surgió la idea, como parte del fortalecimiento institucional necesario, de crear un organismo que desde fuera del poder ejecutivo, y no como parte de él como sucedía con COFETEL, se encargara de dicha tarea.

De esta manera, con la tendencia iniciada en 1993 al dotar de autonomía constitucional al BANXICO, el 11 de junio de 2013 se publicó en el DOF un decreto de reforma constitucional que entre otros artículos enmendó al 28, con lo cual se dio origen a dos nuevos OCAs: la Comisión Federal de Competencia Económica (COFECE) y el IFT. En el caso de este último, lo definió como "un órgano autónomo, con personalidad jurídica y patrimonio propio, que tiene por objeto el desarrollo eficiente de la radiodifusión y las telecomunicaciones, conforme a lo dispuesto en esta Constitución y en los términos que fijen las leyes. Para tal efecto, tendrá a su cargo la regulación, promoción y supervisión del uso, aprovechamiento y explotación del espectro radioeléctrico, las redes y la prestación de los servicios de radiodifusión y telecomunicaciones, así como 
del acceso a infraestructura activa, pasiva y otros insumos esenciales, garantizando lo establecido en los artículos 6o. y 7o. de esta Constitución".

Cabe señalar que tanto la COFECE como el IFT además de haber sido creados en la misma reforma, comparten varios puntos comunes, tanto así que ambas instituciones son autoridades en materia de competencia económica, sin embargo, el segundo lo es exclusivamente para los sectores de radiodifusión y telecomunicaciones al básicamente regular de forma asimétrica a los participantes en estos mercados con el objeto de eliminar eficazmente las barreras a la competencia y la libre concurrencia.

El mismo artículo constitucional, establece como principios rectores del IFT -y también de la COFEGE, en su caso--, entre otros, dos que serán de especial interés en el presente estudio:

1. Dictará sus resoluciones con plena independencia.

2. Podrá emitir disposiciones administrativas de carácter general exclusivamente para el cumplimiento de su función regulatoria en el sector de su competencia.

A través del primero de los principios se reafirma la característica autónoma del IFT, ya que seguramente después de haber pasado por un estadio de órgano desconcentrado de la Secretaría de Comunicaciones y Transportes, se consideró indispensable hacer una separación categórica de dicha dependencia de la Administración Pública Federal (APF). Por lo que hace al segundo de los principios que rigen su actuar, se confirma que como autoridad reguladora, está facultado para emitir disposiciones de carácter general.

Después de conocer los dos principios rectores ya mencionados, se puede colegir que si bien el IFT tuvo una etapa previa que fue la COFETEL (y ésta era parte de la APF centralizada), se entendería que el IFT se trata de una autoridad que tiene una función formalmente administrativa (en parte por su origen en el seno del poder ejecutivo) y, sin embargo, también realiza funciones formalmente 
legislativas y jurisdiccionales; en el primer caso al emitir disposiciones generales y, en el segundo, al dictar resoluciones a controversias planteadas ante sí. Por tanto, estamos ante la presencia de un ente del poder público que lleva a cabo funciones formales que originaria e históricamente han sido ejercidas por el poder Legislativo, por el Ejecutivo y por el Judicial y que, no obstante, no se encuentra adscrito a ninguno de ellos; de ahí su importancia actual y la trascendencia que puede llegar a tener. A diferencia de estos últimos que realizan funciones formales relacionadas con su propia naturaleza y materiales que son características de sus pares, este OCA realiza prácticamente funciones formales de los tres tipos, por mandato constitucional y legal.

\section{ili. Aspectos para considerar aplicables al caso CONCRETO}

En el caso de este ente público, además de la Constitución, son dos las leyes que regulan lo realizado por el mismo, se trata de la Ley Federal de Competencia Económica y de la Ley Federal de Telecomunicaciones y Radiodifusión (LFTR). En ambas leyes se señala que el IFT es la autoridad en materia de competencia económica de los sectores de radiodifusión y telecomunicaciones, ejerciendo en ellas en forma exclusiva las facultades que establecen el artículo 28 de la Constitución, así como ambas leyes. La importancia del IFT como ente regulador se puede ponderar a la luz del peso que la industria de las telecomunicaciones tiene en el crecimiento económico del país. En su "Cuarto Informe Trimestral Estadístico 2016", el IFT señala que el Producto Interno Bruto (PIB) del sector de telecomunicaciones y radiodifusión fue de 546 mil millones de pesos durante el dicho trimestre, con una tasa de crecimiento anual de más del 8\%. Esto resulta en una aportación al PIB nacional del 3.6\%, por lo que la industria de las telecomunicaciones y la radiodifusión ocupó el octavo lugar en términos de las actividades económicas con 
mayor aportación, estando arriba de sector de servicios educativos y por debajo del sector financiero y asegurador (Comunicado de prensa IFT No. 4872017). De igual forma, el IFT señala que en el sector se crearon 273 mil empleos.

En este contexto relativo al peso actual del sector dentro de la economía mexicana, cabe recordar que históricamente han existido en México empresas de las telecomunicaciones y radiodifusión que tomaron gran preponderancia con el paso del tiempo, en algunos casos incluso se llegó a hablar de que se comenzaban a constituir monopolios, los cuales se decidió atacar frontalmente a partir de las reformas en materia de competencia económica y en materia de telecomunicaciones llevadas a cabo en este sexenio. Con dichos cambios, se comenzó a brindarle al Estado Mexicano los instrumentos constitucionales, legales e institucionales para detener la alta concentración que habían logrado tener las empresas participantes de los sectores de telecomunicaciones y radiodifusión, lo que había traído como efecto el desincentivar la competencia en dichos sectores y, así, mantener costos muy altos para los mexicanos.

En aras de generar condiciones económicas tendientes a procurar la competencia dentro del sector, así como incentivar la inversión tanto extranjera como nacional y mejorar los costos para el usuario final, dentro de la reforma fueron incluidos conceptos como el de Agente Económico Preponderante (AEP) y Medidas de Regulación Asimétrica (MRA) por parte del legislador ordinario.

Actualmente, los criterios para ser considerado un AEP pueden encontrarse en la LFTR. De acuerdo con el artículo 262 de dicho ordenamiento, en el caso del AEP se tiene que es cualquiera que cuente directa o indirectamente con más del 50\% de los usuarios suscriptores, audiencia, tráfico o capacidad. La reforma en materia de telecomunicaciones establece, por ejemplo, que:

- Los AEP no podrán cobrar tarifas adicionales por llamadas a teléfonos de otras empresas. 
- Los AEP en telefonía estarán obligados a permitir a sus competidores completar su red para la prestación del servicio de roaming nacional sin costo adicional, a fin de acelerar la cobertura nacional por parte de más prestadores de servicios. ${ }^{4}$

Por lo que hace a las MRA, el propio artículo 262 de la LFTR establece que son las que se imponen para evitar que se afecte la competencia y la libre concurrencia y, con ello, a los usuarios finales. Éstas incluyen en lo aplicable, las relacionadas con información, oferta y calidad de servicios; acuerdos en exclusiva; limitaciones al uso de equipos terminales entre redes; regulación asimétrica en tarifas e infraestructuras de red, incluyendo la desagregación de sus elementos esenciales y; posiblemente, la separación contable, funcional o estructural de dichos agentes.

En lo relativo a ambos conceptos, es el IFT quien de manera exclusiva tendrá la facultad en primer término de declarar AEP a una empresa y, en segundo, de imponerle una MRA. Es el artículo 265 de la LFTR el que establece el procedimiento para resolver una o ambas cosas, resaltando lo siguiente:

1. El IFT notificará al agente de que se trate el proyecto de declaratoria como presunto AEP.

2. El presunto AEP tendrá un plazo de quince días hábiles, contados a partir del día siguiente a aquél en que surta efectos la notificación mencionada en el punto anterior, para que manifieste lo que a su derecho convenga y ofrezca los elementos de prueba que considere necesarios, los cuales deberán estar relacionados con el proyecto de declaratoria de preponderancia.

4 Disponible en línea: <https://www.gob.mx/presidencia/articulos/que-es-un-agenteeconomico -preponderante $>$. 
3. Una vez que comparezca el presunto AEP, el IFT, a través de la autoridad que se determine en su estatuto, se pronunciará sobre la admisión de las pruebas ofrecidas y, en su caso, ordenará abrir un periodo para su preparación y desahogo por un plazo de hasta quince días hábiles.

En caso de que durante la instrucción el IFT considere que es necesario establecer las medidas específicas o asimétricas que se le impondrán al presunto AEP, ordenará su tramitación en vía incidental y resolverá en la definitiva.

En el incidente el presunto AEP manifestará lo que a su derecho convenga respecto de las medidas que, en su caso, se hayan determinado, dentro de un plazo que no excederá de cinco días hábiles contado a partir del día siguiente a aquel en que surta efectos la notificación de la apertura del incidente, pudiendo sólo ofrecer las pruebas que estén directamente relacionadas con las medidas que propone el IFT.

4. El IFT contará con un plazo de cuarenta días hábiles para dictar la resolución definitiva correspondiente, la cual deberá ser notificada dentro de un plazo no mayor a veinte días hábiles contado a partir del día siguiente aquel en que sea emitida por la autoridad correspondiente y, posteriormente, se publicará en el DOF y en la página de internet del propio IFT. 
iv. Determinación como AEP AL GRupo de interés económico del que forman parte América Móvil. S.A.B. de C.V., Teléfonos de México. S.A.B. de C.V., Teléfonos del Noroeste. S.A. de C.V., Radiomóvil Dipsa S.A.B. de C.V., Grupo Carso S.A.B. de C.V., y Grupo Financiero LNBURSA. S.A.B. DE C.V. (TELMEX Y TELCEL) Y MRA IMPUESTAS

Fue a través del ACUERDO P/IFT/EXT/060314/76 de la sesión del pleno del IFT celebrada el 6 de marzo de 2014 que se determinó mediante una resolución de carácter regulatorio como AEP al consorcio del cual forman parte TELMEX y TELCEL, el cual fue previamente determinado como un grupo de interés económico. Cabe señalar que esta determinación fue anterior a la entrada en vigor de la LFTR, la cual como se ha visto, ya establece un procedimiento y criterios detallados para la declaración de un AEP, no obstante dicha determinación de TELMEX y TELCEL como AEP e imposición de MRAs fue hecho con base en las entonces recientes reformas constitucionales en materia de telecomunicaciones y en la entonces vigente Ley Federal de Telecomunicaciones, posteriormente derogada por la LFTR. Es útil señalar que igualmente este nuevo ordenamiento derogó la Ley Federal de Radio y Televisión.

Los resolutivos del Acuerdo en comento señalaron que:

- Se determinara como AEP al grupo de interés económico del que forman parte América Móvil. S.A.B. de C.V., Teléfonos de México. S.A.B. de C.V., Teléfonos del Noroeste. S.A. de C.V., Radiomóvil Dipsa S.A. B. de C.V., Grupo Carso S.A.B. de C.V., y Grupo Financiero lnbursa. S.A.B. de G.V. (TELMEX y TELCEL).

- Se impusiera al AEP anterior, una serie de MRAs necesarias para evitar que se afectara la competencia y la libre concurrencia. En los alcances siguientes, esencialmente: 
- Medidas relacionadas con información, oferta y calidad de servicios, acuerdos en exclusiva, limitaciones al uso de equipos terminales entre redes, regulación asimétrica en tarifas e infraestructura de red, incluyendo la desagregación de sus elementos esenciales y, en su caso, la separación contable, funcional o estructural al AEP en los servicios de telecomunicaciones móviles.

- Medidas relacionadas con información, oferta y calidad de servicios, acuerdos en exclusiva, limitaciones al uso de equipos terminales entre redes, regulación asimétrica en tarifas e infraestructura de red, incluyendo la desagregación de sus elementos esenciales y, en su caso, la separación contable, funcional o estructural al agente económico preponderante en los servicios de telecomunicaciones fijos.

- Medidas en materia de contenidos audiovisuales que se establecerán al AEP en telecomunicaciones.

- Convenio marco de interconexión entre las redes de del integrante correspondiente del AEP con la red pública de telecomunicaciones del concesionario.

- Asimismo, en la resolución el IFT determina medidas orientadas a permitir la desagregación efectiva de la red local fija.

- Se señala que resolución tendría carácter de definitiva, la cual solamente podría ser combatida mediante el juicio de amparo.

Entonces, a través de este Acuerdo se determinó a TELMEX y a TELCEL como AEP y también se les impuso una serie de MRAs destinadas a combatir las barreras a la competencia y la libre concurrencia, así como se dio la posibilidad de permitir la desagregación efectiva de la red local fija. De igual manera, en vista de tratarse de 
una resolución definitiva se señaló que solo podría ser combatida a través del juicio de amparo, es decir, no hay posibilidad alguna de naturaleza recursal ante el mismo IFT, ni ante cualquier tribunal de carácter contencioso-administrativo en forma, por ejemplo, de juicio de nulidad, lo que demuestra la naturaleza formalmente jurisdiccional de esta resolución del IFT.

\section{v. Tarifa Cero en Interconexión, el ACUERdo} P/IFT/2603 14/17Y LA LFTR. LA CLAVE

En el contexto del ACUERDO P/IFT/EXT/060314/76, el IFT con fecha 26 de marzo de 2014 emitió su ACUERDO P/ IFT/260314/17 por medio del cual se determinan "las tarifas asimétricas por los servicios de interconexión que cobrará el agente económico preponderante." Mediante este Acuerdo, el IFT establece una disposición destinada a establecer una MRA orientada a equilibrar la competencia, es decir, la tarifa cero.

Posteriormente con la publicación de la LFTR en el DOF el 14 de julio del año de 2014, se estableció más claramente cuándo una tarifa por servicios de interconexión será asimétrica con el fin de procurar la competencia equilibrada, es así que el segundo párrafo del artículo 131 de la LFTR, conteniendo en lo general los criterios del IFT, establece que siempre que exista un AEP en el sector de las telecomunicaciones o un agente que cuente directamente o indirectamente con una participación nacional mayor al 50\% en el sector, las tarifas de terminación de tráfico fijo y móvil, incluyendo llamadas y mensajes cortos o sms, serán asimétricas conforme a lo siguiente:

1. Los agentes anteriormente referidos, no cobrarán a los demás concesionarios por el tráfico que termine en su red. La llamada "tarifa cero". 
2. Para el tráfico que termine en la red de los demás concesionarios, la tarifa de interconexión será negociada libremente.

Lo anterior se traduce en que si se hace una llamada o envía un mensaje corto con origen en otro concesionario y con destino a TELMEX o TELCEL, estos últimos no cobrarán tarifa alguna, es decir, una tarifa cero. Al contrario, si la llamada o el mensaje corto se originan en TELMEX o TELCEL y terminan en otro concesionario, habrá una tarifa de interconexión negociada entre ellos. Esta asimetría en el trato de unos concesionarios y de otros, busca generar una equidad que paulatinamente combata las barreras a la competencia y la libre concurrencia.

Por tanto, automáticamente al existir un AEP, éste se someterá a estas MRAs sin que medie resolución alguna del IFT u otro ente público, pues simplemente está así establecido en la LFTR, es decir ya existe un mandato legal. De esta manera, lo que alguna vez tuvo que ser resuelto en forma ad hoc por el IFT por medio de una resolución de carácter regulatorio, ahora con lo dispuesto en la LFTR tendría como fundamento la ley misma, obviamente.

Ante esto, se observa que la configuración actual de las MRAs que afectan al AEP en términos de tarifa cero, esencialmente, se desprenden de una resolución de carácter regulatorio por parte del IFT y que posteriormente se contiene en un ordenamiento legal con la publicación de la LFTR; esto demuestra el peso específico que este OCA tiene en el panorama administrativo, normativo y de resolución de controversias en México, al realizar funciones relacionadas de manera formal y en virtud de la fuerza otorgada por la reforma en materia de telecomunicaciones.

Empero, el establecimiento de la tarifa cero en la LFTR por decisión del legislador ordinario encontró en TELMEX y TELGEL a su principal combatiente, al cuestionar la constitucionalidad de esa decisión del Poder Legislativo Federal. 
¿Este ánimo combativo del AEP en contra de la tarifa cero de la LFTR traería consigo la consolidación del IFT como un OCA con fuerza y legitimidad? ¿Se estaba ante un caso paradigmático de los que configuran un sistema normativo e institucional dentro de un Estado?

La respuesta a estas preguntas quedaría en manos de la SCJN.

\section{LA CONSOLIDACIÓN DEL IFT}

Frente a la situación descrita anteriormente, el AEP inicia una lucha frontal en contra de las resoluciones antes descritas y expedidas por el IFT que llevan al establecimiento de una tarifa cero evidentemente perjudiciales para los intereses del primero Asimismo, incluye en ese combate lo establecido al respecto en la LFTR. Es así que el AEP centra su estrategia en tres puntos, haciéndolo por medio de Radiomóvil Dipsa, S.A. de C.V.:

1. Juicio de amparo indirecto en contra del AGUERDO P/ IFT/EXT/060314/76 radicado con el número 42/2014 en el Juzgado Segundo de Distrito en Materia Administrativa especializado en Competencia Económica, Radiodifusión y Telecomunicaciones.

2. Juicio de amparo indirecto en contra del ACUERDO P/ IFT/260314/17 radicado con el número 42/2014 en el Juzgado Segundo de Distrito en Materia Administrativa especializado en Competencia Económica, Radiodifusión y Telecomunicaciones.

3. Juicio de amparo indirecto en contra de (i) la discusión, aprobación y expedición de los artículos 131 párrafo segundo inciso A y párrafo tercero, así como los artículos sexto, vigésimo y trigésimo quinto transitorios de la LFTR, y de la (ii) promulgación del Decreto que contiene las normas impugnadas. Para ello fueron señaladas como autoridades responsables, respectivamente, el Congreso de la Unión y el Presidente 
de la República. Dicho juicio fue radicado bajo el número 204/2014 ante el Juzgado Segundo de Distrito en Materia Administrativa, especializado en Competencia Económica, Radiodifusión y Telecomunicaciones.

En el caso de los dos primeros juicios señalados, ambos fueron sobreseídos y por tanto se negó la protección de la justicia federal. De esta manera la estrategia del AEP se enfocó en el juicio de amparo indirecto 204/2014 en contra de (i) la discusión, aprobación y expedición de los artículos 131 párrafo segundo inciso A y párrafo tercero, así como los artículos sexto, vigésimo y trigésimo quinto transitorios de la LFTR, y de la (ii) promulgación del Decreto que contiene las normas impugnadas.

A la postre, el 18 de febrero de 2015, por sentencia, la protección de la justicia federal sería igualmente negada por el juzgado conocedor del asunto. Sin embargo, es necesario mencionar que de los conceptos de violación mencionados por Radiomóvil Dipsa, S.A. de G.V. destacaban para efectos de este trabajo, los siguientes:

a. El Congreso de la Unión es incompetente para emitir regulación y fijar tarifas de interconexión.

b. El establecimiento del régimen de gratuidad (tarifa cero) viola la garantía de seguridad jurídica por la ilegal extinción del régimen tarifario derivado del Decreto de reforma constitucional.

Es por eso que, ante el recurso de revisión interpuesto por Radiomóvil Dipsa, S.A. de C.V. previa declaración de incompetencia por parte de Tribunal Colegiado de conocimiento por tratarse del análisis de la constitucionalidad de una norma, sería la SCJN quien asumiera su competencia originaria, por lo que el recurso de revisión se tramitaría ante esta última, y se registraría bajo el número 1100/2015. De esta manera se iniciaría el estudio constitucional 
de un asunto que finalmente resultaría paradigmático al fortalecer al IFT y con ello al sistema institucional mexicano recientemente reformado, específicamente le daría fuerza y legitimidad a la figura de los OCAs existentes.

La SCJN, como tribunal constitucional del país, concentró su análisis en el párrafo segundo inciso A y párrafo tercero del artículo 131 de la LFTR, así como en los artículos sexto, vigésimo y trigésimo quinto transitorios del Decreto por el que se expide la LFTR.

El pasado 16 de agosto de 2017, la SCJN pronunciaría su sentencia al amparo en revisión 1100/2015 revocando la sentencia recurrida y otorgando la protección de la justicia federal a Radiomóvil Dipsa, S.A. de C.V., lo que como efecto inmediato generaría la consolidación del IFT como el órgano regulador en materia de telecomunicaciones en México, ya que, grosso modo, el Máximo Tribunal resolvería que el Congreso de la Unión invadió la esfera competencial del IFT al establecer que corresponde al IFT la competencia originaria y exclusiva para fijar el régimen asimétrico relativo a las tarifas de interconexión para la terminación de tráfico en la red móvil del AEP.

La conclusión alcanzada por la SCJN parte del reconocimiento del nuevo diseño institucional que creó al IFT como la instancia especializada en el sector, y que es el organismo que cuenta por tanto con los elementos técnicos para expedir esta regulación, pero sobre todo, con la competencia para ello otorgada directamente por la Constitución.

No obstante se reconocen las facultades constitucionales del Congreso de la Unión para legislar en materia de telecomunicaciones y radiodifusión, esto no implica la sustitución del IFT en el ejercicio de sus propias facultades constitucionalmente establecidas. De esta manera, esta sentencia se convierte en paradigma al consolidar el nuevo diseño institucional que fortalece el papel de los OCAs del Estado, particularmente los encargados de tareas regulatorias. 


\section{vil. Conclusiones}

Dentro del análisis presentado, es importante hacer notar una serie de reflexiones que son necesarias para entender el nuevo paradigma que vivimos, y partimos de la siguiente pregunta: ¿Es esta resolución en favor del IFT una que fortalece, legitima y consolida a los OCAs dentro del sistema institucional mexicano?

Desde mi perspectiva, se tienen distintos elementos para responder a dicha pregunta:

1. Efectivamente se reafirma la fuerza y especialización de los OCAs (no es cosa menor que la doctrina y la jurisprudencia ya consideren que cuentan con una paridad de rango con respecto a los poderes tradicionales), ya que los dota de elementos suficientes y necesarios para hacerse cargo y ser la máxima autoridad en materias especializadas.

2. Se rompe con el paradigma tradicional, lo que hace necesario buscar formas para determinar con mayor claridad la responsabilidad de los OCAs; ya que, en casos como este el IFT no realizó su función y en cambio trasladó de facto su responsabilidad al Legislativo, no cumpliendo con el ejercicio de las facultades exclusivas que le fueron conferidas por la Ley Fundamental.

3. Se deben tener precauciones con este nuevo diseño institucional, principalmente porque podemos incurrir en generar organismos dotados de muchas facultades, pero sin la voluntad o incluso la capacidad de responder de forma eficiente y clara a asuntos que son de su competencia, pudiendo ser omisos al observar que es otro Poder el que está supliendo esa deficiencia.

4. Si bien la SCJN es un órgano fundamentalmente decisor, debe llamar la atención que se ha convertido gradualmente en el Poder que da claridad a la determinación de las facultades 
de los otros; por lo que se puede correr el riesgo de poner en entredicho la paridad jerárquica que debe existir entre ellos. 5. Decisiones como la tomada por la SCJN obligan a redimensionar el fenómeno del poder en México, la irrupción de los OCAs por lo general ha sido causante de una clara reducción en las facultades que puede ejercer el Poder Ejecutivo, y es de esa manera que se ha asumido como algo correcto para de esta manera incrementar y fortalecer los contrapesos existentes frente al último, en un país eminentemente presidencialista. No obstante, la resolución que se analiza tuvo un impacto directo en la línea de flotación del Poder Legislativo, lo que resultó novedoso pues al igual que el Judicial habían resultado los Poderes menos afectados con la llegada de los OCAs, tal vez de ahí pueda entenderse parte de la polémica que generó y que indudablemente se ha convertido en un precedente muy importante.

6. Existe una clara falta de claridad conceptual entre los legisladores y los integrantes de los propios OCAs. Parecería que ahora el Poder Legislativo busca recuperar parte de las facultades que con anterioridad ejercía y las cuales dejo de tener en virtud de reformas constitucionales y legales diseñadas en su seno. No queda duda de que la facultad originaria de regular asimétricamente en tarifas al AEP es única y exclusivamente del IFT, por lo que el Poder Legislativo Federal se extralimitó y elaboró una norma que a todas luces generaría una reducción del margen de maniobra de un ente público que se encuentra al mismo nivel.

7. Los criterios bajo los cuales la SCJN resolvió no son otros que los fijados por el principio de división de poderes, los cuales son establecidos por la Carta Magna. Es por tal razón que consideramos que la teoría tradicional de la división de poderes ha sido rebasada y se está volviendo impostergable 
reconocer que el día de hoy existen diez OCAs que ejercen facultades muy importantes y que agrupados deben ser considerados ya un cuarto poder. La resistencia de parte de la doctrina, del legislador y de los juzgadores a reconocer esta realidad está generando que hoy estas instituciones se encuentren dispersas, dentro de un desorden administrativo, y que el correcto ejercicio de las facultades que les han sido conferidas no sea claro.

8. La sentencia de la SCJN tiene como uno de los ejes alrededor de los cuales se lleva a cabo su argumentación al que llama "Principio de competencia especializada o deferencia técnica", y que ahí mismo se señala "parte del reconocimiento de que las cuestiones técnicas deben ser necesariamente reservadas al Instituto; máxime cuando desde la norma constitucional se advierte, como es en el caso, la existencia de una competencia de carácter originario en materia de regulación asimétrica". Es decir, la exclusividad en las facultades del IFT se debe a dos elementos:

a. Competencia especializada, y

b. Competencia de carácter originario.

Por tanto, se tiene que derivado de la voluntad popular el IFT cuenta con una competencia de carácter originario (toda vez que fue el propio legislador quien reformó la Constitución para determinarlo así) y, además, el hecho de ser un organismo solvente técnicamente para emitir reglas de carácter general y resolver controversias en la materia le brinda la legitimidad suficiente para que el ejercicio de sus facultades exclusivas sea oponible a terceros, los Poderes de la Unión entre ellos.

9. El voto concurrente que formuló el Ministro José Fernando Franco González Salas en el amparo en revisión 1100/2015 fue más allá e hizo una crítica a la resolución aludida al señalar 
lo siguiente: "me separo de las consideraciones de la sentencia en las que se mencionaron lineamientos relacionados con la forma en que el Instituto Federal de Telecomunicaciones debe fijar la tarifa de interconexión, pues considero que ello es contradictorio con la razón central por la que se concedió el amparo, a saber, que el Instituto Federal de Telecomunicaciones es el único órgano constitucionalmente competente para fijar la tarifa de interconexión para los agentes económicos preponderantes". Es decir, ninguno de los tres Poderes deben tener injerencia alguna en los temas que competen únicamente al IFT, pudiendo considerar a éste como un argumento importante para hacerse valer por cualquiera de los nueve OCAs restantes.

10. Actualmente, la fortaleza ganada por los OCAs a partir de algunas de las decisiones tomadas por los propios legisladores y, cuando llegan a presentarse conflictos, por los juzgadores es una realidad; situaciones como aquellas de manera definitiva están reconfigurando la forma y los medios por los cuales el poder es ejercido en México.

Derivado de lo anterior, es importante reconocer que al día de hoy existen en México diez importantes instituciones públicas que en razón de entre otras cosas su creación y configuración señaladas directamente en la Carta Magna, de su competencia especializada, de los importantes temas a los que están dedicadas y de la paridad jerárquica que tienen respecto de los poderes tradicionales, deben ser consideradas un cuarto poder, el cual por cierto se trata de uno constituido. Existen constituciones que ya lo contemplan, como ejemplo se tiene que la Constitución Política de Colombia en su artículo 113 señala lo siguiente:

Artículo 113. Son Ramas del Poder Público, la legislativa, la ejecutiva, y la judicial. 
Además de los órganos que las integran existen otros, autónomos e independientes, para el cumplimiento de las demás funciones del Estado.

Los diferentes órganos del Estado tienen funciones separadas pero colaboran armónicamente para la realización de sus fines.

Con un reconocimiento claro como el señalado anteriormente, se podría comenzar por establecer un orden en esta categoría de entes públicos y de esta manera mitigar el riesgo de que se presenten situaciones indeseables. Finalmente, en resoluciones como las que se han analizado en el presente trabajo, se pueden vislumbrar argumentos a favor de que así sea, a pesar de que aún se pueden notar reticencias francamente endebles. 